\title{
Service innovation from the edges: enhanced by telemedicine decision support
}

\author{
Tonje C. Osmundsen ${ }^{1}$ Ellen A. Andreassen Jaatun ${ }^{2}$ Gunhild Foss Heggem ${ }^{1,3}$ • \\ Bård Erik Kulseng ${ }^{3,4}$
}

Received: 14 December 2014/ Accepted: 26 May 2015/Published online: 21 June 2015

(C) The Author(s) 2015. This article is published with open access at Springerlink.com

\begin{abstract}
Increased prevalence of diabetes creates a need for improved care. Diabetes, as a chronic disease in which the patient is a central asset for effective care, is relevant as an illustrative example for also other chronic diseases that are on the rise globally. Such diseases demand localised co-produced healthcare services that respond to the need for integrated health care. The aim of the current paper was to investigate how telemedicine may facilitate patient consultations that improve quality, efficiency and patient involvement. Patient consultations facilitated by telemedicine were evaluated by the means of qualitative questionnaires to specialist, general practitioner and patients. The questionnaires revealed that the patient consultations improved quality of health care and saved time and cost. Findings suggest that such tripartite consultations may lead to outcomes where patients are empowered to take a more active role, the general practitioner increases his knowledge and competence, and the specialist is able to have a more holistic approach grounded in an understanding of patient history and local context. The support
\end{abstract}

Tonje C. Osmundsen

tonje.osmundsen@samfunn.ntnu.no

1 NTNU Social Research, Dragvoll Allé 38b, 7491 Trondheim, Norway

2 Department of Otorhinolaryngology and Head and Neck Surgery, St. Olavs University Hospital, Trondheim, Norway

3 Center of Obesity Research, St. Olavs University Hospital, Trondheim, Norway

4 Department of Cancer Research and Molecular Medicine, Norwegian University of Science and Technology, Trondheim, Norway of telemedicine strengthens the consultations in terms of synchronous communication with visual clues and reduces time spent on the consultation by the specialist and travel time and cost for the patient. The contribution of this paper is to demonstrate how telemedicine can provide improved patient consultations by altering the established system of healthcare delivery.

Keywords Diabetes - Healthcare delivery ·

Telemedicine $\cdot$ Integrated care $\cdot$ Tripartite joint consultations · Empowerment

\section{Introduction and background}

Diabetes is a disorder that demonstrates the challenges of healthcare systems in most Western countries. Its prevalence is rising sharply [1], and it is a chronic disease which demands close follow-up by both patient and healthcare providers and the treatment options are many and continuously developed.

The rise in type 2 diabetes is related to the increased prevalence and incidence of obesity and particularly affects younger people $[1,2]$. When a patient has diabetes for many years, the risk of complications increases, such as stroke, kidney disease, blindness and amputation. All complications have direct or indirect relations to high blood sugar. Continuous monitoring of blood sugar and correct treatment are of great importance to slow down the progression of the disease and subsequent complications. The traditional treatment offered by the general practitioners (GPs) has, however, not been sufficient, and a great number of patients have not reached the set treatment goals [3]. This has led to more complications in the population and has thus increased the number of patients referred to 
specialist health care. The larger number of patients who are suffering from type 2 diabetes with complications makes it apparent that healthcare services to these patients need to improve, and this concerns both primary and specialist healthcare providers. Efficient treatment needs to be available before complications develop, and in line with national guidelines [4]. This is generally not the case in Norway, or elsewhere [3], as international studies demonstrate a need for improved care for diabetes patients [5-7].

Treatment of diabetes is complicated and demands highly competent care providers, and there are many treatment options $[8,9]$. Care and treatment need to be systematic and up to date on recent research results. The patients themselves need to be highly involved in their own care and treatment, which is demanding in terms of motivation and knowledge. Diabetes is a chronic disease that involves everyday activities, and care decisions need to be taken on a daily basis. The patient needs support and guidance in order to make healthy choices and changes. As continuous care is essential, close collaboration between highly competent care providers and patients is necessary. Studies investigating the role of patient education and involvement indicate positive results for patients who receive training and are involved in their own treatment plan [10]. The challenges apparent in the current health services for diabetes care are also relevant for a range of other diseases and are related to how we organise our healthcare systems. Norwegian health care is a distinct two-level system where specialist healthcare services are owned and financed by the Ministry of Health and Care Services and primary care is organised and financed by the local authorities (municipalities). Primary care includes medical services through GPs as well as nursing home, home care and other services [11-13].

In response to a perceived need to improve the healthcare services and especially the extent of cooperation between levels of healthcare providers, Norway and other countries have instigated political reforms to promote integrated care $[14,15]$. One of the goals of the Norwegian Coordination Reform is to enable the primary care level to assume increased responsibility for prevention and rehabilitation of patients. The goal is to reduce both the amount of referrals to hospitals and the time patients spend at the hospitals. One of the problems facing the Norwegian healthcare system is the insufficient ability of hospitals to absorb patient inflows resulting in long waiting lists [11, $16,17]$.

The main ambition of the Norwegian Coordination Reform [11] is to improve collaboration between and within systems of healthcare providers and to strengthen the capacity and capabilities of the municipal healthcare providers. However, to realise such changes healthcare providers need to find innovative ways for collaboration and strengthen their mutual competence in providing integrated care. While there are different interpretations of such collaboration, here we will use the concept of integrated care [18]. Integrated care may be understood as care that is comprehensive, coordinated, continuous, holistic, empowering, co-produced and collaborative [19].

In this paper, we present a qualitative study focused on the experiences of patients, specialist and general practitioner of patient consultations facilitated by telemedicine. In Sects. 3 and 4, we describe how the use of telemedicine consultations came about and why both healthcare professionals and patients embraced the idea of altering the established system of healthcare delivery. We report on the methods applied in this project in Sect. 5 and the subsequent results from qualitative questionnaires in Sect. 6. Finally, we discuss these results in terms of how they answer prevailing challenges in the healthcare system and the need for patient-centred diabetes care in Sect. 7.

The contributions of our paper are as follows:

We report on the importance of groundwork for enrolling healthcare professionals in primary and specialist health care, patients and their carers, patient organisations, managers and politicians, and the community services at large to achieve successful implementation of telemedicine.

We show why a "bottom-up" approach to service innovation is essential to identify the needs and requirements of healthcare professionals and their patients and to respond to those needs with the implementation of technological solutions.

We present how co-production of services can be achieved, representing a response to challenges in the current organisation of health care.

Our research question is; how can telemedicine facilitate a new form of patient consultation that improves quality, efficiency and patient involvement?

\section{Related work}

A recent review [20] on ICT interventions used to support patients and healthcare professionals in five chronic diseases, including diabetes, revealed that ICTs used for synchronous consultations between patient, general practitioner and specialist are rare. ICTs are most often used for educational or administrative purposes and not linked directly to patient care. For instance, ICTs used towards diabetes patients for consultations ranks low with only two studies found, both of which involve nurses, not doctors. Similar results are found also for other chronic diseases. Another review [21] distinguishes between asynchronous 
and synchronous teleconsultations and interaction. Of the 18 studies identified involving synchronous interaction, most concern healthcare professionals such as nurses, dieticians, physiotherapists and others, interacting with patients for educational purposes. Some studies report on how home telemedicine consultations may replace standard outpatient care [22], where nurses are present at the home of the patients and a specialist participates through videoconferencing. Others report on outreach service delivery from a specialist hospital to regional clinics [23]. However, one study is similar to the one reported in this paper, investigating videoconferencing between specialist, GP and patients [24]. The study, conducted in Austria, investigates transfer of knowledge from specialist to GP by means of telemedicine, in line with a problem-based learning approach with the patient present. Results concerning the effect on the education of the GP are relevant for our study. On the other hand, there is no mention of how patients or specialists perceived the consultation.

The virtual outreach study is a larger study where Harrison et al. [25] explore patient satisfaction with teleconsultations and find that an important distinction may be whether or not the consultation includes a physical examination. Patients were less satisfied with teleconsultations that included a physical examination. Also, patients reported that they sometimes felt excluded from the conversation if the GP and specialist did not take care to involve the patient. These results are relevant for our study.

In a Norwegian context, due to large distances and a highly dispersed population, the northern part of the country has extensive experience with developing and implementing the use of ICTs both as an outreach service from the regional hospital and as a means for coordination between primary and secondary health services. However, there are few studies discussing joint teleconsultations between specialist, GP and patient. Nilsen [26] looks at the exchange of knowledge between the GPs and the specialists in videoconferences and how collaborative work might close knowledge gaps in daily medical practice; however, patients were not present. This is also the case for other studies [27], while it was the GP who was not involved in other studies [28, 29], the exception being a study [30-32] where GPs had received special training to perform endoscopic otolaryngological examinations, which were conducted during a synchronous consultation with a specialist. Patient reported being satisfied with this type of consultation, and it offered the patient new ways of taking part in the consultation.

In sum, few other studies have the same characteristics as the case study reported here, that is, joint consultations between specialist, GP and patient, and synchronous interaction by video conference with the aim to support learning as well as shared treatment decisions. The studies identified were qualitative case studies, and the authors did not find publications on similar quantitative studies.

\section{Case context}

During the past years, the number of consultations of patients with diabetes at St. Olavs University Hospital in Central Norway has increased severely. This is indirectly also a measure of how referrals from GPs to the hospital have increased. From 2010 to 2014, the number of consultations with diabetes patients 16-20 years of age has increased $62 \%$, and for the group between 20 and 44 years of age, consultations have increased $42 \%$. The total number of diabetes consultations at this hospital has increased by $50 \%$ in the same period. A similar increase (53\%) can be found for gestational diabetes, diabetes occurring during pregnancy. Due to a lack of capacity at the hospital, newly referred or pregnant patients are given priority, while routine checks of patients are postponed. This means less patient support and may result in increased risk of medical complications.

As a response to the challenges described above, a Center of Obesity Research (ObeCe) at St. Olavs University Hospital in Central Norway has, since 2005, initiated a broad spectre of projects aimed at improving obesity and diabetes care in the region. They have done so by facilitating increased healthcare literacy in local communities and improved domain knowledge on obesity and diabetes in primary healthcare services. The centre collaborates closely with specialist clinics and primary care providers in the region.

The various projects ObeCe has initiated have involved patients and their organisations as well as stakeholders from healthcare providers, schools and voluntary organisations. The diversity of projects and the large number of stakeholders involved has led to an emphasis on the need for collaboration between health professionals at various levels of the health services. We will mention a few of the projects below to demonstrate how these activities provided fruitful grounds for establishing collaboration between GPs and specialists supported by telemedicine.

Health literacy among the general population in Central Norway was strengthened through a "Healthy Life" campaign focusing on diabetes and overweight. The campaign comprised meetings with stakeholders such as healthcare professionals, community health leaders, politicians, businesses, schools and different voluntary organisations including patient organisations. 
Improvement in knowledge of diabetes and diabetes care among GPs in the region was achieved through a series of local meetings at medical centres and in larger conferences including GPs from across the region. The local meetings were comprised of specialists from the university hospital and GPs and had a problem-based learning approach [33]. Specific patients whom the GP found challenging were discussed, and, in joint deliberations, different treatment options were assessed. Educational positions for GPs in the fields of obesity, overweight and related diseases such as diabetes were established in three hospitals in the region.

Specialised diabetes nurses employed at the hospital have part of their practice in the municipalities, providing knowledge directly to patients, GPs and other health professionals in primary care. Their work contributes to enhancing implementation of guidelines by the GPs, empowering patients with knowledge and close access to the diabetes nurse in their local communities.

All of these activities were essential in improving the health literacy among the local population. They were also important for increasing the knowledge on treatment of obesity and type 2 diabetes among the healthcare providers and for contributing to the implementation of new guidelines for treatment. This led to an increased awareness of the need to improve prevention and treatment of diabetes. A shared understanding of joint responsibility and the need for co-producing integrated services was established between healthcare professionals at the primary and specialist healthcare services.

Healthcare providers from both primary and specialist healthcare services were promoting better collaboration and looked for technological solutions to bridge the physical distance between them. The ambition was that the technological solutions would improve current information exchange (written communication and phone calls) and facilitate faster and more reliable information exchange.

\section{Case study: telemedicine consultations}

In 2013, the telemedicine consultation project, Styrk Video, was carried out in the rural region of Fosen. The region was chosen because patients and healthcare professionals had a shared interest in improving their competence in the prevention and treatment of obesity and diabetes, and had expressed a desire for closer cooperation with the specialist health services. In addition, the clinical setting required quick and continuous decision-making and high-level specialised knowledge for best practice in endocrine disorders. The consultations involved one GP, one specialist in endocrinology and the patient. It was seen as important to improve and support knowledge sharing and decision support between the GP and the specialist, and, at the same time, maintain patient safety and make sure the patients felt well attended to. Telemedicine consultations were seen as a promising tool to support these ambitions.

Independent researchers followed the project closely. They investigated whether the use of telemedicine consultations could meet some of the challenges of achieving improved diabetes care. Below we will report on these findings.

\section{Methods}

Eight telemedicine consultations were evaluated; in seven of these, GP, specialist and patient were present by the means of telemedicine. The patient was in the GP's office together with the GP, while the specialist was at his office at the hospital. One of the consultations was between only the GP and the specialist. The included patients were chosen by the GP, and all of the patients would have been referred to the hospital if the specialist had not been available for consultation through telemedicine. All patients as well as the GP and the specialist were asked whether they agreed to participate in the research project and asked to answer a questionnaire following the consultation.

Author Heggem, an independent researcher, developed the questionnaires in adherence to qualitative methods [34]. The questionnaires were developed in three versions with questions specific for patients, specialist and GP. There were seven questions each to GP and patient, and eight questions to the specialist. The questionnaires included themes such as time spent, perception of the consultation, utility value, comparison to an outpatient consultation, cost savings and responsibility for updating patient records. In all questionnaires, there were open text fields for additional comments related to the questions. Heggem also collected the questionnaires after each consultation, summarised the results and developed categories that summed up the experiences of the participants and the benefits of telemedicine consultations.

The specialist had no access to the GP's electronic health record (EHR) system, only to the hospital EHR, which might contain old notes about the same patient. The consultations lasted an average of $20 \mathrm{~min}$. The GP registered the consultation and the agreed treatment plan in the patient journal. During the consultation, the GP presented the patient's history and the issues to be discussed. Patient, GP and the specialist participated in the discussion. If the medical problem could not be resolved by the telemedicine 
consultation, the patient could thereafter be referred to the hospital. None of the patients included was referred to the hospital since medical problems were resolved in the consultation.

\subsection{Platform}

The telemedicine support platform consisted of a CISCO EX90 TelePresence System at the hospital and a CISCO C40 TelePresence Codec at the GP's office. The system was connected to an encrypted secure line used for health information traffic, provided by HEMIT. ${ }^{1}$ This ensured that all conversations were encrypted end to end and satisfied the norms for information security [35]. This is important when it comes to patient privacy. The login was fast, log history was easy to use, the picture quality was in HD format, and the sound was excellent, according to the participants.

This platform was selected on the basis of costs of investment and interaction with existing computer units. Telemedicine consultations require investments in equipment and maintenance. However, the same equipment can be used for many different purposes where appropriate. This applies to both administrative and patient care tasks. Beyond this, it will not lead to additional costs for the patient, GPs or the specialised health services compared with the current patient care.

\section{Addressing the challenges}

The distinct two-level system of Norwegian health care requires GP and specialist to interact with different financial systems. This entails that when a GP or a specialist has a consultation with a patient, this is paid for by either the municipality or the state. When GPs and specialists consult each other without the presence of a patient, they do not receive payment, either at the municipal level or at the state level. The form of consultation realised in this project, with a patient present, allows both GP and specialist to be compensated financially. This is an important premise for the viability of the project.

\subsection{Patient}

The overall impression was that the patients' experiences of telemedicine consultations were positive. Six out of the seven patients answered that they were "very positive", and the seventh answered "positive". All of the patients (except one with a missing answer) preferred telemedicine

\footnotetext{
${ }^{1}$ HEMIT is the provider of IT services to The Central Norway Regional Health Authority.
}

consultation to being referred to the hospital. All of the patients reported that they were taken good care of and felt safe during the consultation.

The patients experienced that the presence of the GP during the telemedicine consultation with the specialist increased their feeling of security and made it easier for them to understand what the specialist was saying. The patients also commented that they felt it was easier to ask questions in this setting if they did not understand what was being said. One possible reason for this could be that the patients find it easier to ask the specialist questions when the GP is asking questions as well and that the distance between patient and expert is reduced. Another possible explanation is that the patients do not know what questions to ask, but that it becomes clearer when the GP is asking questions to the specialist. The patients involved in these consultations received greater knowledge and understanding of their own disease, and the consultations increased the patient's competence.

Patients travelling from the rural region of Fosen going to the regional hospital will normally spend $1-3 \mathrm{~h}$ on a ferry to reach the hospital. The patients report that they on average saved $2 \mathrm{~h}$ when they had the consultation at their GP's office instead of at the hospital. On average, the patients thus saved 290 NOK (approximately $35 €$ ) in travel costs.

\subsection{Specialist}

The specialist also reported a positive experience from telemedicine consultations. The technical platform was perceived to be user-friendly, and no technical challenges were experienced. The specialist emphasised that telemedicine consultations increase the quality of health care provided to the patients because medical questions can be dealt with in an efficient and secure manner. Patients would otherwise have to wait for an appointment at the hospital, and issues could not be resolved as swiftly. The consultation was more efficient and took less time for the specialist. He did not have to spend time preparing and reading up on the patient history since the GP already had this knowledge readily available. The specialists receive information and opportunities to follow-up, something that may contribute to a more holistic approach towards the patient when specialists realise that treatment needs adaptation to the local environment.

\subsection{General practitioner}

The GP stated that the benefits of using telemedicine consultations were that it was informative and instructive and that he got useful advice on follow-ups and diagnostics. The feedback from the GP shows that telemedicine 
consultations are very well suited for giving general advice and well suited for diagnostics. The GP also feels that such consultations will make him more capable of treating other similar patients independently, thus reducing the need to consult a specialist.

There was an added value in having the patient present at the consultation. The GP was actively engaged in problem-solving with the help of both specialist and patient, something that strengthened the learning process. The alternative would have been to refer the patient to the hospital and be informed of the results later on. Here, the GP could engage in problem-based learning and get immediate feedback.

The use of telemedicine consultations reduced the number of referrals from primary to specialist health services. Before the consultation, the GP was planning to refer all of the patients to the hospital; however, none of the patients in this study were referred after the consultation. Although the number of participants included in this project is small, it gives some indications of the cost-benefits of such consultations.

\subsection{An efficient model}

Both specialist and GP experience teleconsultations as efficient when compared to the established model of healthcare delivery based on referrals and sequential consultations. The figure below illustrates the workflow in these two different models. It shows how the established model requires repeated cycles to achieve quality information between the two doctors and the patient.
It may be argued that in a telemedicine consultation, two doctors are occupied at the same time, and the GP could have treated someone else while the specialist saw the patient. However, the workflow in the established model is overly complicated, and all parties spend time on receiving, interpreting, understanding and verifying information from others. In a video consultation, patient and doctors are present to provide and correct any information simultaneously. The grounds for treatment decisions are transparent and accessible to all involved, especially to the patient. Another outcome of the video consultation is the long-term added benefit of learning between those present. At the outset, the GP did not feel capable of treating these patients, but he states after the consultations that he is more capable to treat similar patients in the future, thus reducing the need for referrals to the hospital.

The questionnaires explored the range of possible benefits of joint consultations. The case study show that improved communication, learning, efficiency and empowerment are the perceived benefits. The results suggest that all the involved parties had a positive experience from the consultations; this also includes their experience with the use of the technology.

\section{Discussion}

Telemedicine can facilitate consultations between patient, GP and specialist, resulting in co-production of healthcare service. The consultations applied here is a new construct we would like to refer to as a tripartite consultation. The
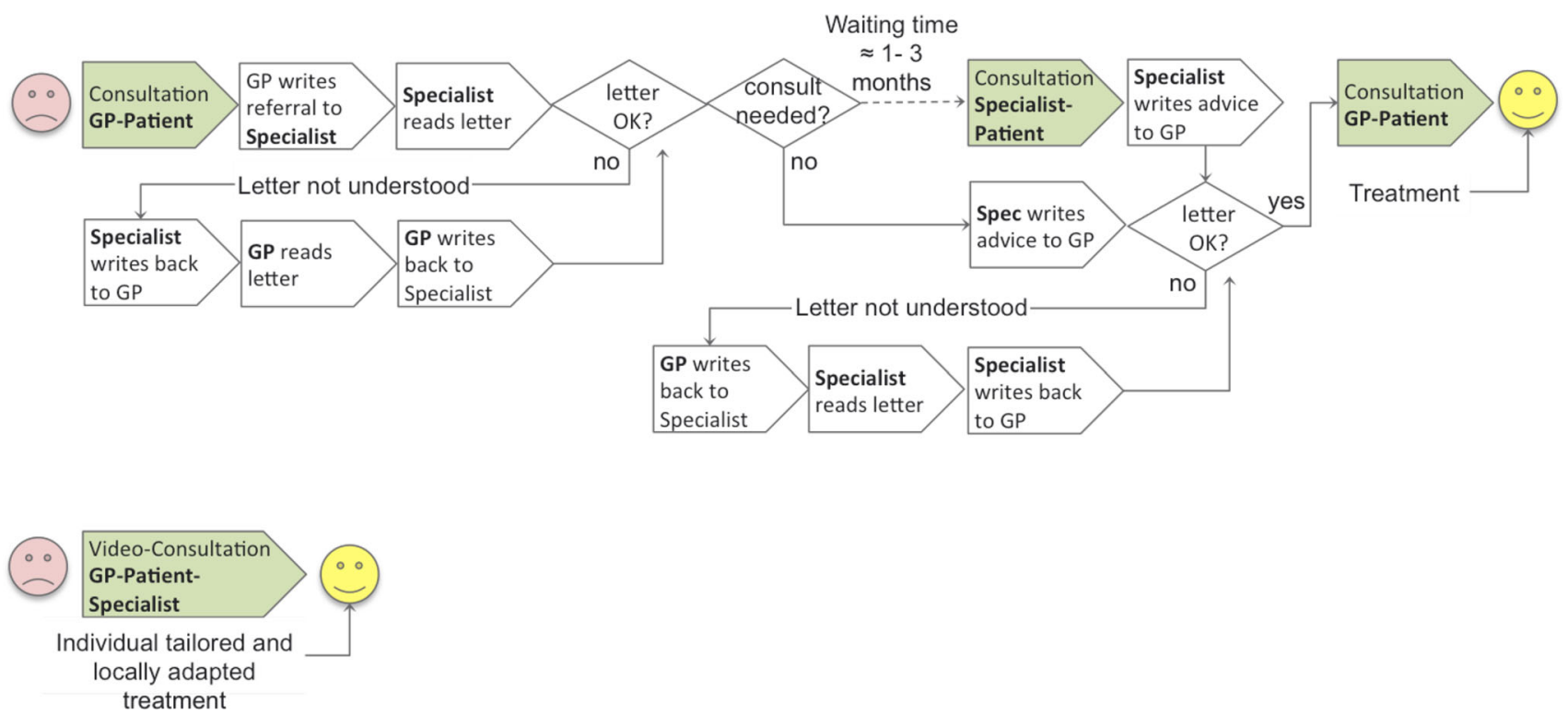

Fig. 1 Workflow in traditional versus telemedicine consultations 
consultation includes three participants (GP, specialist and patient with diabetes). All participants have their specific role and are equally important. Each participant provides information from his or her field of expertise (e.g. blood sugar measurement device, patient record, self-assessment, local knowledge and disease-specific knowledge), and through dialogue, they reach a consensus for treatment.

Co-production of healthcare delivery differs from mainstream organisation of health care in several areas. It combines the expertise of both specialist and GP, situating the treatment decisions closer to the patients' local environment and local health providers. Additionally, traditional health care is based on a sequential model of delivery where GPs and specialist form separate decisions in their own fields of expertise, see Fig. 1. In this project, the GP and specialist are able to form joint decisions grounded in their respective expertise, something that allows for a more holistic approach. In a tripartite consultation, patients are involved and are empowered to ask for more information, enabling them to influence choices concerning their own health. Other studies also find that patient experiences telemedicine consultations as positive $[25,30-32]$. To have the patient present in a tripartite consultation was important to enhance the learning process for all involved.

The tripartite consultation has potential to provide benefits at three levels of healthcare delivery:

- Patient: Better diabetes control and reported confidence in his or hers healthcare providers.

- GP: Empowerment and more experience in the field of diabetes treatment through problem-based learning.

- Specialist: Quality control of treatment delivery outside of hospital. Reduced time spent on consultations. Receiving information on structure and associated problems in the local community that can be applied to improve support to the local community in future work.

Furthermore, the interaction and information exchange between levels of health care are traditionally based on asynchronous media such as letters or electronic messages between healthcare providers. The written communication between healthcare providers may be tarnished by misunderstandings and lack of information, as illustrated in Fig. 1. Martinussen [36] reports how specialists often consider referral letters from GPs to specialists unnecessary. The specialists believed that the patient could have received satisfactory care from the primary healthcare service. A similar study [37] revealed that only half of the referral letters in the sample contained satisfactory written information and this can pose a risk to patients. A complementary study on community nurses shows that nurses felt the information from specialist health care was incomplete and did not provide the information they needed to do their work [38]. When using telemedicine consultations, the benefits of synchronous communication with visual control allows the participants to immediately respond to information and ensure that others have a shared understanding. The decision-making is based on discussions with all parties and can be immediately corrected if a common understanding is not achieved. The process has many common features to social media as defined by Kaplan and Haenlein [39].

Diabetes treatment requires individual tailoring that demands accurate data. The treatment goal is to reduce side effects such as hypoglycaemia and prevention of comorbidity. Both problems are dependent on correct interpretation of patient-generated data from self-measurement and laboratory results. The shift in diabetes prevalence and occurrence in younger patients has been challenging for GPs [40, 41]. Utilisation of available data produced by patients (blood sugar measurement) for better tailoring of treatment requires both a change in workflow and increased knowledge. In order to optimise the treatment, local knowledge and a close relation to the patient and the patient's network are important. This places the GP in a key position to facilitate better treatment and supports greater involvement of patients.

The tripartite consultation developed in this project is efficient and responds to current needs to improve diabetes care. For specialist health care, increased diabetes prevalence in younger patients has put great pressure on the available resources. Telemedicine consultation reduces the time spent by the specialist on each patient and empowers the GP to treat more patients at his practice without having to refer them to the hospital. This is also been shown in other studies [24, 42]. The patient saves travel costs and time. Norway is a country with a distributed population and travel distances to the nearest specialist care provider may be considerable. For patients living in rural areas, this may result in high costs due to travel expenses and work absence.

Furthermore, as chronic diseases such as diabetes affect everyday life and demand close follow-up, support from a healthcare provider in close proximity is important [43, 44]. Specialist healthcare providers may lack important information regarding facilities and local parameters for treating the patient. In order to tailor the treatment and follow-up of patients living in rural areas, better communication support is required. In the rural region of Fosen, a broad range of projects providing increased health literacy to the local population and to healthcare providers allowed the telemedicine consultations to be successfully implemented. The need to strengthen diabetes care had been made apparent to those involved and created an enthusiasm towards developing improved solutions for delivering health care. The Styrk Video project was tailored to a rural 
area where the resources are scarce compared to urban areas, but the enthusiasm and motivation of the local inhabitants to improve health and well-being may be higher. High-quality health care may be taken for granted in an urban area, but may not be a matter of course in more rural areas. The tripartite consultation represents a new construct in both rural and urban areas, but can be perceived differently in the two areas. The consultations was perceived as a positive addition to already existing services in the rural area, since the alternative still existed, but implied spending most of the day travelling to the hospital. In an urban area, such consultation might be perceived as a substitute for the existing services provided by the specialist health care without offering any added benefits. In terms of service innovation, it might therefore be better to develop and test such new solutions in a rural area, where the need for services is most apparent. Also, service innovation in areas with limited resources and lack of qualified personnel may be beneficial due to the constant need for tailoring and finding new creative solutions, adapted to the local context.

A bottom-up approach to service innovation has shown to be a more sustainable solution than a top-down approach [45]. The initiative to change the system as well as a framework for implementing changes started within the organisation. The participants were motivated by the perceived needs and also received immediate reward for their efforts in terms of increased knowledge. A bottom-up approach to service innovation can thus support team building and make different groups pull in the same direction for a common goal. The utilisation of tools and devices (telemedicine) was not the main purpose, but the need for rapid turn taking and visual control for more efficient communication made the telemedicine consultations and thus a tripartite solution possible.

We note that a limitation of this study is that the number of participants is low, but the answers from all involved were consistent, and they were also in accordance with results from other studies done on telemedicine in the healthcare sector [25]. However, the concept should be refined and tested in a larger population and with more GPs and specialists.

\section{Conclusion}

For patients with chronic diseases, continuous care and follow-up by medical personnel from both primary and secondary care are essential. The co-production of healthcare services provides integrated care that is holistic, comprehensive and empowering for the patient. Improving healthcare services through co-production between specialist and primary healthcare providers also has relevance for other types of diseases, especially lifestyle related and chronic diseases. Co-production merges the specific fields of expertise of separate healthcare professionals and allows for a more holistic approach to healthcare delivery.

Telemedicine provided a platform for shared consultation that escaped the usual traps of misunderstandings grounded in written communication between specialist and GP. Healthcare professionals are trained in oral communication, something telemedicine supports. The consultations provided benefits for those involved in terms of competence, decision support and involvement, and provided improved efficiency and quality for the healthcare services. On the other hand, tripartite joint consultations challenge the established models of healthcare delivery between primary and specialist health care by crossing organisational boundaries supported by different financial structures. This makes it difficult to establish new practices, even though the healthcare professionals may be in favour of change. This may also explain why joint consultations are not in more widespread use.

Service innovation needs to respond to challenges felt by healthcare professionals. The telemedicine equipment used in this project is merely a tool to realise effective consultations between professionals who feel they need closer collaboration, both to increase their own competence and to provide integrated care to chronically ill patients. Future studies should investigate how telemedicine tripartite consultations could involve also other healthcare providers, and in other fields besides diabetes.

Acknowledgments The Central Norway Regional Health Authority funded the research project and the telemedicine equipment. Parts of the analysis and writing of the paper were included in a project evaluating the Coordination Reform in Norway funded by The Research Council of Norway (Number 229623). We would like to thank all informants for participating in the study. We would also like to thank our anonymous reviewers for helpful advice and comments.

Open Access This article is distributed under the terms of the Creative Commons Attribution 4.0 International License (http://creativecommons.org/licenses/by/4.0/), which permits unrestricted use, distribution, and reproduction in any medium, provided you give appropriate credit to the original author(s) and the source, provide a link to the Creative Commons license, and indicate if changes were made.

\section{References}

1. Midthjell K, Krüger O, Holmen J, Tverdal A, Claudi T, Bjørndal A, Magnus P (1999) Rapid changes in the prevalence of obesity and known diabetes in an adult Norwegian population. The NordTrøndelag Health Surveys: 1984-1986 and 1995-1997. Diabetes Care 22:1813-1820

2. Danaei G, Finucane MM, Lu Y, Singh GM, Cowan MJ, Paciorek CJ, Lin JK, Farzadfar F, Khang Y-H, Stevens GA et al (2011) National, regional, and global trends in fasting plasma glucose 
and diabetes prevalence since 1980: systematic analysis of health examination surveys and epidemiological studies with 370 country-years and 2-7 million participants. Lancet 378:31-40

3. Collinsworth A, Vulimiri M, Snead C, Walton J (2014) Community health workers in primary care practice redesigning health care delivery systems to extend and improve diabetes care in underserved populations. Health Promot Pract 15:51S-61S. doi:10.1177/1524839914539961

4. Haas L, Maryniuk M, Beck J, Cox CE, Duker P, Edwards L, Fisher EB, Hanson L, Kent D, Kolb L, McLaughlin S, Orzeck E, Piette JD, Rhinehart AS, Rothman R, Sklaroff S, Tomky D, Youssef G, Force on behalf of the 2012 SRT (2013) National standards for diabetes self-management education and support. Diabetes Care 36:S100-S108. doi:10.2337/dc13-S100

5. Aschner P, LaSalle J, McGill M, on behalf of the Global Partnership for Effective Diabetes Management (2007) The team approach to diabetes management: partnering with patients. Int $\mathrm{J}$ Clin Pract 61:22-30. doi:10.1111/j.1742-1241.2007.01606.x

6. Pill R, Stott NC, Rollnick SR, Rees M (1998) A randomized controlled trial of an intervention designed to improve the care given in general practice to Type II diabetic patients: patient outcomes and professional ability to change behaviour. Fam Pract 15:229-235. doi:10.1093/fampra/15.3.229

7. Van den Arend IJ, Stolk R, Krans HM, Grobbee D, Schrijvers AJ (2000) Management of type 2 diabetes: a challenge for patient and physician. Patient Educ Couns 40:187-194. doi:10.1016/ S0738-3991(99)00067-1

8. Amed S, Nuernberger K, Reimer K, Krueger H, Aydede SK, Ayers D, Collet J-P (2014) Care delivery in youth with type 2 diabetes-are we meeting clinical practice guidelines? Pediatr Diabetes 15:477-483. doi:10.1111/pedi.12147

9. Cefalu WT (2013) "TODAY" reflects on the changing "Faces" of type 2 diabetes. Diabetes Care 36:1732-1734. doi:10.2337/ dc13-0765

10. Fitzner K, Greenwood D, Payne H, Thomson J, Vukovljak L, McCulloch A, Specker JE (2008) An assessment of patient education and self-management in diabetes disease management-two case studies. Popul Health Manag 11:329-340. doi:10.1089/pop.2008.0012

11. Van den Noord P, Hagen T, Iversen T (1998) The Norwegian health care system. Organisation for Economic Co-operation and Development, Paris

12. Helles $\varnothing$ R, Fagermoen MS (2010) Cultural diversity between hospital and community nurses: implications for continuity of care. Int J Integr Care 10:e036

13. Paulsen B, Romøren TI, Grimsmo A (2013) A collaborative chain out of phase. Int J Integr Care 13:e008

14. Norwegian Ministry of Health and Care Services (2009) The coordination reform. Proper treatment-at the right place and right time. Oslo: Norwegian Ministry of Health and Care Services; [Summary in English]. Full version in Norwegian: Report no. 47 (2008-2009) to the storting

15. Mur-Veeman I, van Raak A, Paulus A (2008) Comparing integrated care policy in Europe: does policy matter? Health Policy 85:172-183. doi:10.1016/j.healthpol.2007.07.008

16. Byrkjeflot H, Neby S (2004) The decentralized path challenged? Nordic health care reforms in comparison. Stein Rokkan Centre for Social Studies. http://www.ub.uib.no/elpub/rokkan/N/N02-04. pdf

17. Siciliani L, Hurst J (2005) Tackling excessive waiting times for elective surgery: a comparative analysis of policies in 12 OECD countries. Health Policy 72:201-215. doi:10.1016/j.healthpol. 2004.07.003

18. Kodner DL, Spreeuwenberg C (2002) Integrated care: meaning, logic, applications, and implications-a discussion paper. Int $\mathbf{J}$ Integr Care 2:e12
19. Ferrer L, Goodwin N (2014) What are the principles that underpin integrated care? Int J Integr Care 14:e037

20. Wildevuur SE, Simonse LWL (2015) Information and communication technology-enabled person-centered care for the "big five" chronic conditions: scoping review. J Med Internet Res 17:e77. doi:10.2196/jmir.3687

21. Verhoeven F, Tanja-Dijkstra K, Nijland N, Eysenbach G, van Gemert-Pijnen L (2010) Asynchronous and synchronous teleconsultation for diabetes care: a systematic literature review. J Diabetes Sci Technol 4:666-684

22. Whitlock WL, Brown A, Moore K, Pavliscsak H, Dingbaum A, Lacefield D, Buker K, Xenakis S (2000) Telemedicine improved diabetic management. Mil Med 165:579-584

23. Smith AC, Batch J, Lang E, Wootton R (2003) The use of online health techniques to assist with the delivery of specialist paediatric diabetes services in Queensland. J Telemed Telecare 9(Suppl 2):S54-S57. doi:10.1258/135763303322596273

24. Abrahamian H, Schueller A, Mauler H, Prager R, Irsigler K (2002) Transfer of knowledge from the specialist to the generalist by videoconferencing: effect on diabetes care. J Telemed Telecare 8:350-355. doi:10.1258/135763302320939248

25. Harrison R, MacFarlane A, Murray E, Wallace P (2006) Patients' perceptions of joint teleconsultations: a qualitative evaluation. Health Expect 9:81-90. doi:10.1111/j.1369-7625.2006.00368.x

26. Lundvoll Nilsen L (2011) Collaborative work by using videoconferencing: opportunities for learning in daily medical practice. Qual Health Res 21:1147-1158. doi:10.1177/1049732311405683

27. Donnem T, Ervik B, Magnussen K, Andersen S, Pastow D, Andreassen S, Nørstad T, Helbekkmo N, Bremnes RM, Nordoy T (2012) Bridging the distance: a prospective tele-oncology study in Northern Norway. Support Care Cancer 20:2097-2103. doi:10. 1007/s00520-011-1319-1

28. Rotvold G-H, Knarvik U, Johansen MA, Fossen K (2003) Telemedicine screening for diabetic retinopathy: staff and patient satisfaction. J Telemed Telecare 9:109-113. doi:10.1258/ 135763303321327984

29. Moseng D (2000) Teledermatology-experiences from Northern Norway. Tidsskr Nor Laegeforen 120:1893-1895

30. Pedersen S, Hartviksen G, Haga D (1994) Teleconsultation of patients with otorhinolaryngologic conditions. A telendoscopic pilot study. Arch Otolaryngol Head Neck Surg 120:133-136

31. Pedersen S, Holand U (1995) Tele-endoscopic otorhinolaryngological examination: preliminary study of patient satisfaction. Telemed J 1:47-52

32. Holtan A (1998) Patient reactions to specialist telemedicine consultations-a sociological approach. J Telemed Telecare 4: 206-213

33. Neville AJ (2009) Problem-based learning and medical education forty years on. Med Princ Pract 18:1-9. doi:10.1159/000163038

34. Silverman D (2009) Doing qualitative research, 3rd edn. SAGE Publications Ltd, Thousand Oaks

35. Directorate of Health (2014) Code of conduct for information security. The healthcare and care services sector. https://ehelse. no/code-of-conduct

36. Martinussen PE (2013) Referral quality and the cooperation between hospital physicians and general practice: the role of physician and primary care factors. Scand J Public Health 41:874-882. doi:10.1177/1403494813498951

37. Garåsen H, Johnsen R (2007) The quality of communication about older patients between hospital physicians and general practitioners: a panel study assessment. BMC Health Serv Res 7:133. doi:10.1186/1472-6963-7-133

38. Melby L, Hellesø R (2010) Electronic exchange of discharge summaries between hospital and municipal care from health personnel's perspectives. Int J Integr Care 10:e39 
39. Kaplan AM, Haenlein M (2010) Users of the world, unite! The challenges and opportunities of social media. Bus Horiz 53:59-68. doi:10.1016/j.bushor.2009.09.003

40. Song SH (2014) Young-onset type 2 diabetes: no room for complacency. Lancet Diabetes Endocrinol 2:924-925. doi:10. 1016/S2213-8587(14)70147-0

41. Narasimhan S, Weinstock R (2014) Youth-onset type 2 diabetes mellitus: lessons learned from the TODAY study. Mayo Clin Proc 89:806-816. doi:10.1016/j.mayocp.2014.01.009

42. Nilsen LL (2012) Collaboration between professionals: the use of videoconferencing for delivering E-health. Future Internet 4:362-371. doi:10.3390/fi4020362

43. Clark NM, Quinn M, Dodge JA, Nelson BW (2014) Alliance system and policy change necessary ingredients for improvement in diabetes care and reduction of disparities. Health Promot Pract 15:11S-22S. doi:10.1177/1524839914543829

44. Lewis MA, Bann CM, Karns SA, Hobbs CL, Holt S, Brenner J, Fleming N, Johnson P, Langwell K, Peek ME, Burton JA, Hoerger TJ, Clark NM, Kamerow DB (2014) Cross-site evaluation of the alliance to reduce disparities in diabetes clinical and patient-reported outcomes. Health Promot Pract 15:92S-102S. doi: $10.1177 / 1524839914545168$

45. Cresswell K, Sheikh A (2009) The NHS care record service (NHS CRS): recommendations from the literature on successful implementation and adoption. Inform Prim Care 17:153-160 